\title{
Documentation and investigation of missing health care equipment: The need to safeguard high priced devices in health care institutions
}

Amanda Cheung ${ }^{1}$, Nancy Clayden ${ }^{1}$, Wrechelle Ocampo ${ }^{1}$, Linet Kiplagat ${ }^{1}$, Jaime Kaufman ${ }^{1}$, Barry Baylis ${ }^{1,2,3,4}$, John M. Conly $1,3,4,5$, William A. Ghali ${ }^{1,2,3,4,6}$, Chester H. Ho ${ }^{1,7}$, Henry T. Stelfox ${ }^{1,3,4,6,8,9}$, David B. Hogan ${ }^{* 1,3,4,6,10}$

${ }^{1}$ W21C Research and Innovation Centre, Cumming School of Medicine, GD01 Teaching Research \& Wellness Building, University of Calgary, Calgary, AB, Canada

${ }^{2}$ Division of General Internal Medicine, Cumming School of Medicine, University of Calgary, Calgary, AB, Canada

${ }^{3} \mathrm{O}^{\prime}$ Brien Institute for Public Health, University of Calgary, Calgary, AB, Canada

${ }^{4}$ Department of Medicine, Cumming School of Medicine, University of Calgary, Calgary, AB, Canada

${ }^{5}$ Snyder Institute for Chronic Diseases, Cumming School of Medicine, University of Calgary, Calgary, AB, Canada

${ }^{6}$ Department of Community Health Sciences, Cumming School of Medicine, University of Calgary, Calgary, AB, Canada

${ }^{7}$ Division of Physical Medicine \& Rehabilitation, Department of Clinical Neurosciences, Foothills Hospital, University of Calgary, $A B$, Canada

${ }^{8}$ Alberta Health Services, $A B$, Canada

${ }^{9}$ Department of Critical Care Medicine, Cumming School of Medicine, University of Calgary, Calgary, AB, Canada

${ }^{10}$ Division of Geriatric Medicine, Department of Medicine, University of Calgary, Calgary, AB, Canada

Received: December 16, 2016

DOI: $10.5430 /$ jha.v6n2p10
Accepted: February 8, 2017

Online Published: February 14, 2017

\begin{abstract}
Objective: Missing medical equipment in health care settings decrease productivity, increase spending to replace losses, and potentially endanger patient safety. By documenting and investigating the causes of these incidents, strategies to prevent future occurrences can be developed.

Methods: As an example of this approach, we describe the inadvertent disposal of an expensive medical device during a randomized controlled trial (RCT) conducted within a medical facility. The incident was carefully documented and investigated shortly after it occurred. This information was used to develop targeted interventions to prevent further occurrences.

Results: The device was a mattress overlay connected to a computer monitor that generated a continuous pressure image for use by nursing staff in the prevention of pressure injuries. An Environmental Services staff member disposed of one of these devices when the room of an enrolled patient was cleaned following their transfer to another unit. Miscommunication (or a misunderstanding of communicated information) and lack of awareness were identified as the main causes of this error.

Discussion: By using the loss as a learning opportunity, the investigation of the incident led to strategies for preventing future occurrences. These included frequent training sessions for staff and improvements in signage. A detailed, factual and timely investigation of the events around the loss of armamentarium coupled with analysis on how to prevent future occurrences should be considered for all incidents involving high cost equipment.

Conclusions: A standardized, non-judgmental approach to documenting and investigating the causes of costly equipment loss can lead to strategies for improved asset management and the prevention of further incidents of this nature.
\end{abstract}

Key Words: Misplaced, Missing, Medical equipment, Medical device, Case report, Continuous pressure mapping

*Correspondence: David B. Hogan; Email: dhogan@ucalgary.ca; Address: HSC-3330 Hospital Dr. NW, Calgary, AB T2N 4N1, Canada. 


\section{INTRODUCTION}

The capital expenditure for machinery and equipment in the health and social care sector in Canada was 3.2 billion CAD in 2014. ${ }^{[1]}$ As healthcare devices become increasingly complex and ubiquitous, national spending on them is expected to increase. Consequently, hospitals are looking for opportunities to better manage necessary and costly equipment. This would include addressing unanticipated losses from misplaced, inappropriately discarded, and/or stolen equipment.

The impact of unanticipated losses can decrease productivity, increase spending to cover replacement costs, and potentially endanger patient safety. Studies have shown that nurses spend over 20 minutes per shift searching for misplaced medical equipment, which takes time away from caring for patients. ${ }^{[2-5]}$ Coustasse et al. ${ }^{[6]}$ reported that in 2004 the annual cost of lost and stolen equipment was estimated as 4,000 USD per hospital bed. The cumulative cost of replacing missing high-priced items such as ventilators and electrocardiograms was reported at 300,000 to 400,000 USD for two American hospitals per year. ${ }^{[7]}$

While publications specifically on misplaced hospital items are limited ${ }^{[8-10]}$ studies on errors in healthcare settings indicate that drawing attention to these mishaps assists in heightening awareness of their occurrences among managers and staff. ${ }^{[8]}$ Investigations can identify the causes of lost equipment and inform equipment management strategies. According to Tucker and Sitkin, the best approach to minimize operational failures, such as lost medical equipment, would be understanding their causes linked with implementing solutions to identified issues. ${ }^{[8,11]}$ Documenting and exploring the etiology of these events can serve as a valuable learning opportunity for staff to implement systems-level improvements ${ }^{[12]}$ that would decrease future occurrences and assist in reducing costs.

We describe the loss of an expensive medical device during a randomized controlled trial (RCT) conducted in a tertiary care teaching hospital in Calgary, Alberta, Canada. We use our case to propose an approach to investigating, reporting, and implementing targeted solutions to reduce costs, prevent future losses, and improve service quality. While this report deals with equipment being used for clinical research, as noted previously, loss of expensive equipment occurs regularly in hospitals. ${ }^{[7,13,14]}$

\section{METHODS}

Immediately following the discovery of the loss of an expensive medical device being used in the conduct of an on-going clinical trial, a Research Associate (RA) affiliated with the study conducted a detailed, factual, non-judgmental, and timely investigation of the events around the incident. Findings of the investigation were analyzed to develop targeted interventions for the prevention of future occurrences. The approach used was purposefully modeled on the case reporting done for patient injuries or "near misses" in a hospital setting.

The definition of health care (or medical) equipment used for this paper is a device used in the treatment, mitigation, diagnosis or prevention of a disease or abnormal physical condition. ${ }^{[15]}$

\section{RESUlTS \\ 3.1 Case history}

The missing equipment was part of a continuous pressure imaging device that was being studied in hospitalized patients at risk for pressure injuries (see NCT02325388 at ClinicalTrials .gov). ${ }^{[16]}$ The Conjoint Health Research Ethics Board at the University of Calgary approved the study (REB13-0794). The study was conducted on five separate nursing units in a tertiary care hospital and involved the use of an electronic medical device with individual sensors embedded within a durable, non-permeable, thin, flexible mattress overlay that was designed to measure and provide a visual image of interface pressure. Each mattress overlay system cost approximately 15,000 CAD. Once placed over a bed mattress, the device is connected to a computer monitor to generate a continuous profile of interface pressure between the bed and the patient's body. The mattress overlay was intended to be used sequentially on multiple study participants during the course of the RCT. Unit staff, including unit managers, registered nurses, licensed practical nurses, nursing assistants, and unit clerks involved in the study, were offered in-service training sessions given by the research staff on study purpose, protocol, and device management and storage. The unit staff were trained to remove the mattress overlay, computer monitor and mounting pole from an enrolled patient's room and store the device in a designated storage area if a study participant expired, was discharged or transferred from the unit unexpectedly after hours or before the end of the three-day study period. All study materials were clearly labelled with contact information for research staff who were available by mobile telephone and email 24 hours a day, seven days a week to answer any questions regarding study participants, study equipment, and the protocol.

A 90-year-old female patient admitted to hospital with pneumonia was enrolled into the study after giving informed consent. At the time of her enrollment, the patient was being cared for on Unit A, a unit involved in the trial for over four months that had already recruited more than 50 study participants. The study RA fitted the mattress overlay over 
the bed mattress with a sheet placed on top of the overlay as per product directions and connected it to the computer monitor located on a pole near the head of the bed. As per study protocol, the mattress overlay was to be left on the patient's mattress for three consecutive days.

At 04:05 hours of the second study day, the patient was transferred to Unit B. The patient's original bed remained in her room on Unit A (the patient was transferred to Unit B on a stretcher). The patient passed away later that morning. An Environmental Services staff member cleaned the patient's room on Unit $A$ at 04:25 hours subsequent to the transfer of the patient. The study RA was not contacted or notified about the patient's transfer and subsequent passing at the time.

Later in the morning, the study RA visited Unit A to monitor the equipment. Nursing staff on Unit A informed the RA of the patient's transfer and subsequent death on Unit B. The computer monitor and pole were found in the designated storage area, but the mattress overlay was missing. Inquiries of nursing staff regarding the location of the mattress overlay were unrewarding on both units. To locate the missing overlay the RA spoke with nursing staff, thoroughly searched both Units A and B, contacted morgue staff, and communicated with linen service to ask about the overlay. These attempts were unsuccessful in locating the missing item. The RA then contacted the Environmental Services housekeeping services supervisor to determine if the person who cleaned the room on Unit A had seen the mattress overlay. The Environmental Services supervisor indicated that the Environmental Services staff member who cleaned the room had no recollection of a mattress overlay in the room. The RA re-checked and did a final search on the units before asking to speak directly with the Environmental Services staff member. Once the RA described in detail the mattress overlay, the Environmental Services staff member recalled seeing the overlay in the patient's room when it was cleaned. The Environmental Services staff member then indicated that they placed the mattress overlay into a large garbage bag that was sent to the hospital incinerator, thinking that the item was waste.

\subsection{Case analysis}

According to the Environmental Services protocol for discharge/transfer cleaning of isolation rooms in this hospital, all medical/technical equipment must be removed from the room by the unit staff before Environmental Services staff begin cleaning. ${ }^{[17]}$ If any unusual items are still in the room, the Environmental Services staff are to notify nursing staff. During the day, each unit has a dedicated Environmental Services team. Overnight, on-call Environmental Services staff are dispatched as needed to service the more than 1,000 hospital patient rooms and other areas throughout the facility. Calibration and maintenance (including periodic checks) of medical equipment is managed by the Clinical Engineering Department. They are responsible for an inventory count using a barcoding system at our facility. However, when medical equipment goes missing, responsibility for searching and documenting the losses was unclear and varied between units. This was evident in investigating the loss of our mattress overlay. It was challenging to identify a point of contact regarding missing equipment. No internal incident reporting system had been implemented on the units involved in our study to document lost equipment. These system-level issues at our facility were identified as areas that needed to be addressed. Discussions about organizational process and responsibility for missing equipment inquiries are on-going. The adequacy of staff training about our research study was also explored. Initially training sessions were only provided to nursing staff working on the units participating in our study. Following our investigation, we recognized that equipment management is multidisciplinary and involves hospital staff beyond nursing personnel. It was concluded that deficiencies in ensuring that all staff working on the participating units received, understood and complied with training on study procedures had arisen. The primary cause of the incident was felt to be inadequate training of Environmental Services staff (in particular, the rotating nighttime staff) and miscommunication between unit and Environmental Services staff providing coverage that night. This led to a lack of awareness of how to handle the medical device used in our study. In order to resolve this issue, our research staff organized and provided additional training sessions to unit and Environmental Services staff that included casual, evening, night and weekend employees. The training sessions provided an overview of our research study and specific information on the mattress overlay device and the correct protocol to follow when dealing with a room containing the device. Improvements in signage, including photographs of the equipment, were created to assist staff in recognizing the device. Additional resources, including a concise and simple information sheet on the study and the device, were provided to the Environmental Services department for training newly hired members on the topic. Ongoing training sessions for Environmental Services department will be provided every six months (or as needed) in addition to annual training sessions for unit staff for the duration of the study.

\section{Discussion}

With the increasing use of often costly medical devices in the delivery of health care, hospitals have developed methods to manage this limited resource. These include ways to identify losses from misplaced, inappropriately discarded, 
and/or stolen equipment. The available literature on the approaches used is scarce. ${ }^{[6]}$ Inventory counts, barcoding systems and Radio Frequency Identifications (RFID) have been the most frequently reported. ${ }^{[6]}$ Implementation challenges that can present barriers to their uptake can occur, and while they can help in identifying losses they do not directly address their underlying causes. Inventory reports are often performed retrospectively and only provide estimates of cost and quantity of equipment lost. With barcoding, each piece of equipment must be manually scanned and reconciled with the inventory. ${ }^{[6]}$ These methods are generally not useful in understanding the events leading to the losses and generating targeted solutions. The determination of loss may only occur after the equipment has been missing for some time. Radio Frequency Identification technology appears to be a more promising approach to the timely identification of losses by providing real-time locating feedback for tagged-items that have gone missing. ${ }^{[6]}$ However, its implementation in health care is still fairly new with only $10 \%$ of American hospitals using this technology as of 2013. ${ }^{[6]}$ Concerns regarding RFID that may influence its acceptance include the significant implementation cost, uncertain return of investment, organizational challenges in integrating it within pre-existing facility practices, user compliance, and issues of information security. ${ }^{[6,18,19]}$ Its use may also not prevent future equipment losses if it is not based on an investigation of the events leading to the loss. A detailed, factual and timely investigation of the events around the loss coupled with analysis on how to prevent similar incidents is required to minimize future occurrences. ${ }^{[8]}$

The inadvertent misplacement and subsequent disposal of the mattress overlay was an unfortunate, unanticipated and costly mishap in our RCT. Acknowledging the loss as a learn- ing opportunity, the cause was thoroughly investigated and the results were used to generate targeted strategies to prevent future occurrences. These included frequent training sessions for staff and improvements in signage. The documentation and investigation of the causes of instances of missing expensive medical equipment could and should lead to organizational changes. By understanding the chain of events, issues resulting in lost equipment can be identified and improved on systematically. ${ }^{[8]}$ Documenting and sharing information regarding the loss of valued medical equipment can be useful in identifying similarities both within and between healthcare organizations that could be linked to pre-emptive strategies for loss prevention. We propose that a consistent approach, which could include the use of a standardized reporting instrument, to investigate, document, and analyze lost equipment above a certain cost threshold be implemented by health care facilities. While the threshold for defining high cost for a medical device will vary based on geography, setting, and over time, a suggested one for North American hospitals at this time is greater than 5,000 USD. ${ }^{[20]}$ Similar to traditional case reporting on injuries and "near misses" in a hospital setting, reports on missing equipment should be investigated and documented in a non-judgmental and factual fashion. The investigation and report are in no way designed to lay blame, rather, it should aim to understand the systems issues and to determine ways to improve policies and procedures to prevent future occurrences. To effectively implement this approach, identifying a designated individual or team to investigate and document the events leading up to the loss of equipment is essential. There also has to be a commitment to act on the results of the investigation. Recommendations of possible strategies for mitigating and managing equipment loss as learned from this case are presented in Table 1.

Table 1. Recommendations for the investigation of the loss of costly hospital equipment

\begin{tabular}{l}
\hline Recommendation \\
- $\begin{array}{l}\text { Prior to implementing new equipment, institutions and organizations should reduce the risk of equipment loss through } \\
\text { pre-emptive risk assessments and strategies }\end{array}$ \\
- Identify a responsible party (person or unit) for investigating and reporting on the loss of medical equipment above an \\
established cost threshold (e.g., greater than $\$ 5,000 \mathrm{USD}^{[20]}$ ) \\
- Ensure the identity of this person or unit is widely known within the hospital as well as the standardized process that is to be \\
used for identifying and referring equipment losses \\
- The identified responsible party conducts a thorough investigation of events leading to the loss immediately following its \\
discovery - the focus is on identifying causes and proposing solutions, not assigning blame \\
- A report summarizing the findings of the investigation and recommendations made for preventing future losses is prepared \\
and provided to the stakeholder groups within the hospital in a manner that protects the confidentiality of the involved parties \\
Recommendations to prevent future losses will typically entail staff training and education (e.g., in-servicing new employees \\
regarding equipment cost and maintenance, periodic educational updates for current employees) \\
On an annual basis a summary of activities relating to the extent and cost of lost equipment and recommendation to minimize \\
it is prepared and shared/monitored within the organization
\end{tabular}




\section{Conclusions}

Health care facilities should consider establishing a formal process to investigate incidents where costly medical equipment has been lost. This would consist of a thorough investigation of events relating to the loss of missing device or equipment in order to both identify the primary cause(s) and generate targeted solutions for prevention of future occurrences. Collating and disseminating these reports would assist in drawing attention to key recurring issues within and across health care facilities, which could provide a platform to improve asset management.

\section{ACKNOWLEDGEMENTS}

We would like to thank the unit staff and the Environmental Services department, who supported this report and assisted with the additional in-service training sessions. We would also like to thank the Alberta Innovates Health Solutions (AIHS) Collaborative Research and Innovation Opportunities (CRIO) team grant (number 20130152) for funding and supporting this report.

\section{CONFlicts of InTEREST Disclosure}

The authors declare they have no conflict of interest.

\section{REFERENCES}

[1] Statistics Canada. [Internet]. Capital expenditures for machinery and equipment by sector, by province and territory (Canada). Statistics Canada. 26 February 2014. [cited 2016 April 20]. Available from: http://www.statcan.gc.ca/tables-tableaux/sum-s om/101/cst01/busi02a-eng.htm

[2] Buyurgan N, Lehlou N. Portable Asset Management in Hospitals. Systems Analysis Tools for Better Health Care Delivery. 2013; 21-36. https ://doi.org/10.1007/978-1-4614-5094-8_2

[3] Oh J. Case Study: Implementing RTLS at Texoma Medical Center [Internet]. 2010 November 17. Available from: http://www. beckershospitalreview.com/hospital-man agement-administration/case-study-implementing-rtl s-at-texoma-medical-center.html

[4] Potter P, Boxerman S, Wolf L, et al. Mapping the Nursing Process. Journal of Nursing Administration. 2004; 34(3): 101-109. PMid: 14770070. https://doi.org/10.1097/00005110-200402000 $-00009$

[5] Tucker A, Spear SJ. Operational Failures and Interruptions in Hospital Nursing. Health Services Research Journal. 2006; 41(3): 643 662. PMid: 16704505. https://doi.org/10.1111/j.1475-6 $773.2006 .00502 . x$

[6] Coustasse A, Tomblin S, Slack C. Impact of Radio-Frequency Identification (RFID) Technologies on the Hospital Supply Chain: A Literature Review. Perspectives in Health Information Management. 2013; 10: 1-17.

[7] Rosencrance L. [Internet]. Boston hospital will track assets with wireless system. IT World Canada. 2004 September. [cited 2015 October 22]. Available from: http://www.itworldcanada.com/article/boston-hos pital-will-track-assets-with-wireless-system/17325

[8] Tucker A. The impact of operational failures on hospital nurses and their patients. Journal of Operations Management. 2004; 22: 151-169. https://doi.org/10.1016/j.jom.2003.12.006

[9] Dare F. [Internet]. The High Cost of Nurses' Communication Challenges: Cisco Survey Report. Cisco Internet Business Solutions Group (IBSG). 2009 September. [cited 2015 November 4]. Available from: https://www.cisco.com/web/about/ac79/docs/Nurs es_Survey_Report_0923FINAL.pdf

[10] Gurses A, Carayon P. Performance obstacles of intensive care nurses. Nursing Research. 2007; 56(3): 185-194. PMid: 17495574. https://doi.org/10.1097/01.NNR.0000270028.75112.00
[11] Sitkin S. Learning through failure: the strategy of small losses. Research in Organizational Behavior. 1992; 14: 231-266.

[12] Woolever DR. The Impact of a Patient Safety Program on Medical Error Reporting. Advances in Patient Safety. 2005; 1: 307-316.

[13] Shaban B, Paredes D. [Internet]. Taxpayers Spent Over \$11 Million on Hospital Equipment That's Now Missing. 2015 November 18. [cited 2016 January 29]. Available from: http://www.nbcbayarea.com/investigations/Santa-Cla ra-Valley-Medical-Center-Missing-Equipment-Taxpaye rs-Money-351196811.html

[14] Johnson C. [Internet]. Lost medical gear drains DHBs. 2013 June 9. [cited 2016 January 29]. Available from: http: //www .nzherald.c o.nz/nz/news/article.cfm?c_id=1\&objectid=10889289

[15] Health Canada. [Internet]. Drugs and Health Products: Medical Devices. Health Canada. 2012 August 28. [cited 2017 Feb 2]. Available from: http://www.hc-sc.gc.ca/dhp-mps/md-im/inde $\mathrm{x}$-eng.php

[16] Wong H, Kaufman J, Baylis B, et al. Efficacy of a pressure-sensing mattress cover system for reducing interface pressure: study protocol for a randomized controlled trial. Trials. 2015; 16: 1-11. PMid: 26420303. https ://doi.org/10.1186/s13063-015-0949-x

[17] Alberat Health Services. Alberta Health Services Practice Support Document Protocol. [Internet]. 2016 January 30. [cited 2016 March 23]. Available from: http: //www.albertahealthservices.ca/assets/healthinfo/i pc/if-hp-ipc-les-discharge-transfer-isolation.pdf

[18] Fisher JA, Monahan T. Evaluation of real-time location systems in their hospital contexts. International Journal of Medical Informatics. 2012; 81: 705-712. PMid: 22857790. https://doi.org/10.101 6/j.ijmedinf.2012.07.001

[19] Okoniewska B, Graham A, Gavrilova M, et al. Multidimensional evaluation of a radio frequency identification wi-fi location tracking system in an acute-care hospital setting. Journal of the American Medical Informatics Association. 2012; 19(4): 674-679. PMid: 22298566 https://doi.org/10.1136/amiajnl-2011-000560

[20] Okike K, O'Toole R, Pollak A, et al. Survey Finds Few Orthopedic Surgeons Know The Costs of The Devices They Implant. Health Affairs. 2014; 33(1): 103-109. PMid: 24395941. https: //doi.org/10.1377/hlthaff.2013.0453 